${ }^{1}$ Department of Collective Health, Health Sciences Center, State University of Londrina, Londrina, Paraná, Brazil.

${ }^{2}$ Departament of Oral Medicine and Pediatrics Dentistry, Health Sciences Center, State University of Londrina, Londrina, Paraná, Brazil.
Corresponding author:

Prof. Dr. Pablo Guilherme Caldarelli Curso de Odontologia - Unidade Campus - Rod. Celso Garcia Cid PR445 - KM 380 - Londrina Paraná, Brasil

Contato: Fone (43) 3371-6742

E-mail: pablocaldarelli@uel.br

Received: June 09, 2020

Accepted: August 26, 2020

\section{The Dental Specialties Center as a point of attention in the Oral Health Care Network in a region of Paraná}

\author{
Alessandra de Oliveira Lippert ${ }^{1}{ }^{1}$, Fernanda de Freitas \\ Mendonça ${ }^{1}$ (D), Brígida Gimenez Carvalho ${ }^{1}$ (D), Pablo \\ Guilherme Caldarelli2,* (D)
}

The National Oral Health Policy (NOHP) arose as an alternative for the improvement of oral healthcare to all Brazilians. On the perspective of specialized care, Dental Specialties Centers (DSC) were implemented. Aim: The aim of this manuscript was the analysis the role of the DSC for the organization of the Oral Health Care Network $(\mathrm{OHCN})$ in the $20^{\text {th }}$ Regional Health Section (RHS) of Paraná. Methods: It is a qualitative study, which analyzed the role performed by the regional DSC Type III, a reference for 18 municipalities. The data were collected through interviews carried out in the period between March and April 2019. Fourteen professional dentist-surgeons, nine from Basic Health Units (BHU) and five from the DSC were interviewed. For data analysis, speech analysis was used. Results: An active role of the regional DSC was identified in this healthcare region, as it acts as a fundamental point in the strengthening of the network, in the process of regionalization and in the integrality of care. Conclusions: However, the importance of broadening the access to specialized care needs to be highlighted, to guarantee the continuity of care started at the $\mathrm{BHU}$.

Keywords: Comprehensive health care. Dental care. Health policy. Oral health. 


\section{Introduction}

Previously to the National Oral Health Policy (NOHP), also known as "Smiling Brazil", the dental care in public services was restricted almost exclusively to the basic services provided to certain age groups in the population, with a large restrained demand ${ }^{1}$. These services were in a high extent inefficient, underfinanced and were not supported by the necessary political will2. Data from the Ambulatory Information System of the National Health System (NHS) (in Portuguese, "SIA/SUS") in 2002, indicated that specialized services did not correspond to more than 3,5\% of the total dental procedures ${ }^{3}$. The low capacity to offer specialized attention services was evident, and it was undermined, so, consequently, the whole dental care and the establishment of adequate reference and counter reference systems in oral health in almost all regional health systems ${ }^{4}$.

Due to this scenario, the guidelines of the NOHP were established in 2004, with the proposal of reorganizing oral health in all levels of care ${ }^{4}$. The guidelines had as their main goal to overcome the inequalities brought by the logic imposed by the hegemonic Market Odontology and the history of withdrawal and lack of commitment with the oral health of the population ${ }^{5}$. These change initiatives aimed to qualify the practices and services offered, through a series of actions in oral health, including universal access and integral assistance in oral health ${ }^{6}$

On the perspective of access to specialized dental services in the scope of the $\mathrm{NHS}$, the strategy adopted by the Ministry of Health $(\mathrm{MH})$ was the implementation of Dental Specialties Centers (DSC) ${ }^{2,7}$. The NOHP presents a model of attention which advocates that patients assisted at the Basic Health Units (BHU) should be sent to DSC when necessary, in order to assure the integrality of assistance, aiming, in this way, to overcome the fragmentation of actions and discontinuity of health care ${ }^{8,9}$.

Hence, the DSC should be implemented in all regions, according to municipal and regional health plans in each state ${ }^{3,10}$. In the regionalization logics, they must be present in a number which has to be proportional to the local population, territorial characteristics and articulated with the offer of basic procedures forming a network of oral healthcare ${ }^{11}$.

In 2010, a macro institutional proposal of organization of the NHS arises, with Health Care Networks $(\mathrm{HCN})$, coordinated by the Basic Care $(\mathrm{BC})^{12}$. In the oral health field, the organization of the systems according to the HCN model is favored by the actual design of NOHP, which was delineated by the principles of care and integrality ${ }^{13}$. In Paraná, the Oral Health Care Network (OHCN) was implemented in 2014, as a public policy for the state, and it was considered the sixth priority $\mathrm{HCN}^{10,14}$.

Due to this model, the DSC are configured as attention sites with specificities defined in the field of the dental specialties, but they need to be integrated with the network of services they belong to ${ }^{15}$. Hence, the aim of this manuscript was to analyze the role performed by a DSC in the organization of the OHCN in a regional health section of the State of Paraná 


\section{Materials and methods}

It is a qualitative study, which analyzed the regional DSC Type III bound to Intermunicipal Health Consortium of the West Coast of Paraná (Ciscopar). This DSC is a referral in the specialized dental care at the $20^{\text {th }}$ Regional Health Section (RHS) to the teams of $\mathrm{BHU}$ in the 18 municipalities in the consortium, with a population of about four hundred thousand inhabitants, and it is implemented in the municipality of Toledo ${ }^{16}$, as shown in Picture 1.

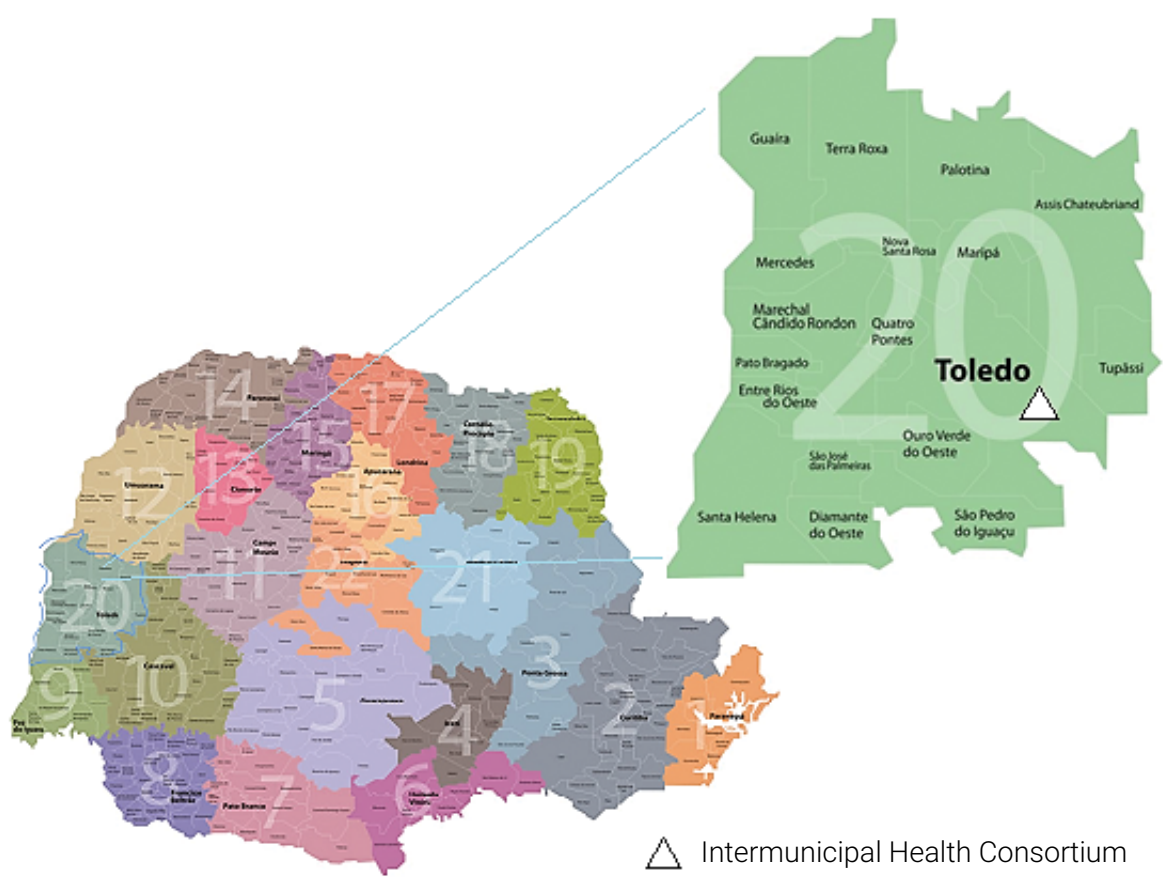

Source: Paraná State Department of Health ${ }^{16}$, adapted by the authors.

Picture 1. Political map of the $20^{\text {th }}$ RHS and location of regional DSC type III.

From all the headquarters municipality, Toledo, is considered of large size (above one hundred thousand inhabitants), five others are classified as small size II (between twenty thousand and one hundred thousand inhabitants) and twelve are considered small size municipalities I (up to twenty thousand inhabitants). Regarding the socioeconomical figures, according to the Institute of Economical and Social Development of Paraná (IPARDES), the region presented indexes ranging above 0,6000, which demonstrates medium and high performance conditions ${ }^{17}$.

The participants in the study were 14 professional dentist-surgeons, nine from a $\mathrm{BHU}$ and five from a DSC, and were considered key informants in the study. In high complexity, there were not any participants, because there was no contact with the referral hospital. The data collection happened through interviews oriented by a semi-structured script and were performed between March and April in 2019, contain- 
ing the following questions related to the role of the CEO in RASB and the relationships established with the other levels of care, especially to AB: 1. For you, what is the DSC's role in the oral health network? 2. What are the relationships established with the other levels of care, especially primary care?

The interviews were recorded, transcribed, and analyzed by the analysis of the speech from Martins and Bicudo ${ }^{18}$. First, the ideographic analysis was performed through fluctuating reading of the speeches and then meaning units (MU), words or sentences which have sense on the perspective of the studied phenomenon. In the following, the interpretation of the $\mathrm{MU}$ was performed and the convergence of the $\mathrm{MU}$ inside the speech of each interviewee. Finally, the nomothetic analysis was conducted by re-reading the MUs and identifying the convergences and divergences, making the construction of categories of analysis possible. The process of coding occurred so that the initials CD were attributed to the professional dentist-surgeons. The professionals of the $\mathrm{BHU}$ received a numerical sequence from 1 to 9 (CD1 to CD9) and those from the DSC, from 10 to 14 (CD10 to CD14), and they were numbered according to the order of the interviews, in order to assure their confidentiality. The recordings were eliminated after the transcription.

The ethical aspects were strictly followed according to Resolution Nr. 466/2012 which rules the research involving human beings ${ }^{19}$. The study was submitted and approved by the Committee on Ethics and Research of the University that the researchers are linked to, with the opinion number 3.120.681 and CAAE 04165518.2.0000.5231.

\section{Results}

Regarding the specialized oral health, in 2018, State of Paraná relied on 53 DSC in 42 municipalities. The analyzed region in the present study has only one DSC, implemented in the municipality of Toledo ${ }^{10,16}$.

This DSC is a type III, with regional range, and is managed by an Intermunicipal Health Consortium (IHC), and it favors the process of regionalization and the offer of medium complexity services in a hierarchical manner. It is a reference to specialized dental care on the specialties of minor oral surgery in hard and soft tissues, oral diagnostics with emphasis in diagnostics and detection of oral cancer, specialized periodontics, endodontics, assistance to patients with special needs, Pediatric dentistry, as well as total and partially- removable dental prosthesis.

The service has nine dentist-surgeons to assist these specialties, as well as three assistants and three technicians in oral health, and it is open 40 hours weekly. There is the Regional Laboratory of Dental Prosthesis (RLDP) connected to the DSC, which makes total prosthesis and partial removable prosthesis for the patients of the NHS.

In the perception of the BHU teams, the main role of the DSC consists in performing the specialized procedures and in supplying the demands and needs of BC. For us, it is extremely important to have a specialized assistance, but the role itself, is to supply our needs here (at the BHU), the dentists (female) are not in condition to perform these services (CD5). 
It must be emphasized that the DSC offers services the BHU does not include, with an "affordable price" to the municipalities in the consortium, because sometimes [...] we at the $B C$ are not able to make, for example, a prosthesis (CD7). In addition, there is a financial advantage in a DSC of regional range, administered by the $\mathrm{IHC}$. According to the highlight:

The municipalities would not be able to afford private or outsourced services, because the value the DSC charges is much lower. It is a particularly good work for the municipalities, I believe it is an excellent support with good cost as well (CD7).

Most of the professionals, 11 out of the 14 questioned, from the BHU or from the DSC, recognize how important the role of the DSC beyond the offer of specialized care is. As a specialized care site, it collaborates in the problem-solving ability of the network, supports BC, and contributes for the continuity of the dental assistance and the integrality of care. Thus, the DSC is considered an important complement to give sequence to the treatment, and without it, the specialized care would not happen, and extractions and dental losses would increase (CD2).

Hence, specialized services also contribute for the maintenance of teeth and mutilation reduction, and in the offer of procedures, that, previously, could only be performed at the private network. If there was not the DSC, many patients would end up by having to perform extractions, and not an endodontic treatment, and if there is no place to send (the patient) to a root canal treatment, so the extraction will be indicated (CD4).

The DSC has a fundamental role, almost everything the patient needs, we can solve. And the population comes here (to the DSC), we are not taking them from private Odontology, because they would not be able to afford it, so there is also a social role (CD13).

Regarding the performance of the BHU professionals, they report they are able to supply "basic needs" of the user, so that with the help (of DSC), in more complicated, delicate cases, the offered treatment ends up by being more resolutive, and this contributes for the integrality, the care (CD5).

The professionals also report they have confidence to refer the user, because they believe they will have their treatment completed. They observe the efficiency and problem-solving capacity of the DSC, so that they develop dependence on its services.

Moreover, actions were identified, some more, others less institutionalized, in the sense of diagnostics support, matrix support, and shared actions, performed with the aim of improving the interface between BC and the DSC. As exemplified in the $\mathrm{BHU}$ professional speech, about the meetings performed to implement a ruling protocol in oral health:

Yes, the protocol meetings are particularly important, because the specialties are being trained, what we need to know to provide the basic care. This help you there (at the DSC), for us to refer the patient in a better way and gives us a better view to act (CD1).

A good communication between the levels was observed in the testimonies which were collected previously. I see a very good dialogue, between the BHU and the DSC 
(CD7). A very calm relationship (CD6). However, the case discussion practice in the teams is still incipient, there is a lack of it, the support does not occur the way I think it should (CD9). Some reservation is done regarding the close dialogue among the technical teams themselves:

The administrative seeks more contact than necessarily the technical area because there could be a partnership, a closer relationship among the technical teams. That is because, as I have already said in a meeting, we are not enemies, we work together (CD12).

The access occurs by a mechanism of reference starting at the $\mathrm{BHU}$, as the DSC has issued the counter references, which are used as important ways of information among the levels of care. Therefore, this mechanism is accomplishing the role of maintaining communication among the levels of care. However, during presentation, the limiting factors are unclear and not objective information are given, besides the adopted strategy of a printed counter reference form, relying on the user to take the document, which is considered the information "messenger."

We cannot let only the patient to be responsible for bringing the information. There should be a mean of communication, via computer, or a list, to which the municipalities could refer "that patient's treatment is over, the prosthesis was made", for them to be able to perform an active search, in order to see if that treatment was finished, or whether it was lost (CD10).

The scheduling of appointments at the DSC is performed by a computerized system, on which the municipal scheduling sets directly a consultation for the desired specialty, which was requested by the dentist-surgeon from the $\mathrm{BC}$ on the reference form. The amount of vacancies is determined by means of a quota distribution among the municipalities in the consortium, according to the population of each them. The system has been shown practical for the users and has optimized the work process. However, it can favor the distancing among the teams because when we were able to talk, it was easier, now everything is scheduled by the system (CD6).

Regarding the restrained demand for the specialties, the largest lines were found by the experts of Endodontics and Dental Prosthesis. The last one had an average waiting time of about three years. Our line has just above a thousand patients for prosthesis, we are calling patients from 2016 (CD9).

We have difficulties in the referral due to the quantity of municipalities the DSC assists, we finish the treatment here, and we send them to an endo, or a prosthesis, which are the most requested specialties and it takes a longer time (CD6).

Finally, when the patients were questioned about the quality of the services that were performed at the DSC, the professionals stated that the services are done in a satisfactory way, with a good technical quality. According to them, is something expected from specialists (CD14).

\section{Discussion}

Analyzing the socioeconomical situation of a region is a way to understand the social and economic development of the municipalities which belong to it ${ }^{20}$. Some authors 
verify that the relation of the Human Development Index (HDI) influences the performance of the $\mathrm{DSC}^{3,21}$ proportionally. The higher the HDI, the better the performance evaluations of the units ${ }^{3,22}$. Thereby, the socioeconomical figures must be considered to analyze the role performed by the DSC, as they can be influenced by contextual local characteristics of the sites they are in.

The organization of the OHCN at the $20^{\text {th }} \mathrm{RHS}$ through the implementation of the DSC of regional range, supports the studies which corroborates that in Brazil 90,4\% of the DSC are located in medium complexity reference areas in Odontology ${ }^{3,22}$. According to the Directing Plans of Regionalization, the regional reference centers are considered landmarks for the structure of a $\mathrm{OHCN}^{19}$. Moreover, the authors of a conducted study in the State of Maranhão, claim that municipal DSC do not work as regional references and weaken the process of regionalization, as they are distributed in an uneven way in the territory, and indicate a low offer of specialized care services ${ }^{23}$.

As regards to the maintaining entity of the DSC, the Report of $1^{\text {st }}$ Cycle of External Assessment of the National Program for the Improvement of Access and Quality of the DSC (NPIA - DSC) shows that the second main manner of hiring dentist-surgeons is through $\mathrm{IHC}^{24}$. The State of Paraná has proposed actions to improve the specialized care in the state, by incentivizing the implementation of new DSC with regional range, as well as a financial incentive to $\mathrm{IHC}$ to improve assistance in already installed units ${ }^{25}$.

This information reinforces the speech of the professionals which says the regional DSC and/or those administered by IHC, for some time, show themselves more resolutive and economical for the municipalities. This organization favors the conformation of regionalized networks, by considering the their principles and hierarchy, so that the process of de-concentration does not overload the municipalities, and the essential conditions like economic efficiency and rational use of the existing resources are kept $\mathrm{t}^{11,26,27}$.

The results of this study agree with those of other authors, as they affirm that the DSC were planned to shelter the typical medium complexity demands of oral care, organically referred by $\mathrm{BHU}^{8}$. It is observed this is the gateway for the system, care regulator, and its professionals act in the screening and generation of demand by motivating the referral to specialized care.

However, it is important to highlight the professionals' dependence of the BHU, regarding the services of the DSC. The external assessors of the NPIA-DSC observed that many DSC end up absorbing the demand of the BC, so that many times, the professionals of the BHU limit themselves to perform some urgency procedures and the continuity of the treatment is performed by the medium complexity professionals 8,24 .

Therefore, the medium complexity would be exposed to perform BC typical procedures, deviating from its central objective. Despite the implementation of a DSC in municipalities where BC is not properly structured, it is not recommended ${ }^{28}$. This can certainly be the cause of restrained demand and overload of specialized services, as far as the effective work of the DSC depends on a proper interface.

Besides, a service network mistakenly centered at the DSC has a low-resolution potential and transfers it to the full role of care in oral health. The NOHP signs that the 
favorable environment to the integral approach is found in the BHU scenarios and not in the DSC, whose methods still work based on procedures, and is not able to absorb all oral health needs of the population.

However, gaining access to such services for the population is extremely important, as it allows the continuity of dental treatments. Before NOHP, specialized treatments were generally available in the private sector, being expensive and inaccessible. For this reason, the structure of specialized care through the implantation of the DSC, allowed the flow of procedures with greater technological density and continuing assistance to the user, without breaking the line of oral health care ${ }^{19}$.

The restrained demand to the DSC is compromising to the integrality of care. Dental Prosthesis and Endodontics also appear with bigger restrained demand in another study ${ }^{29}$. The authors observe that the broadening of dental health actions at the $\mathrm{BHU}$ increased the demand for specialized treatment, and the DSC are not able to absorb it properly ${ }^{29}$. Another compromising factor which is causative of restrained demand is, despite the large number, the distribution of the DSC in Paraná is still disproportionate ${ }^{30}$.

It is up to the DSC as well, to execute matrix support actions and permanent health education, in a regional and macroregional basis, by sharing responsibilities with the other care points in the network ${ }^{10,23}$. The matrix support is a fundamental component to the integration among services, it favors a better interface and provides the effective work and the improvement in the quality of care. The existence of proper articulation between the $\mathrm{BHU}$ and specialized care is indispensable, guided by the premises which direct the regulation of access to healthcare in the regional arrangements $s^{9,24}$.

Proximity processes are fundamental among the teams, with incentive to discuss the actions to be implemented and for the use of management instruments, such as protocols, for example ${ }^{15}$. In addition, the investment of the management on the capacitation of dentist-surgeons and teams with emphasis on the use of these protocols could be able to generate positive impacts in the integrality of oral healthcare ${ }^{8}$.

It was verified that this DSC promotes matrix support actions, but case discussion is incipient. A study which was conducted in Paraná revealed that in only one DSC $(2 \%)$ in the state, the matrix support was verified ${ }^{30}$. Such incoherence among the results can be justified by the periods of carry out, so that this study is based on the Report of the $1^{\text {st }}$ Cycle of External Assessments of NPIA-DSC, performed in 2013. In this way, it can be presumed there have been progress in the development of matrix support actions.

The referral to the DSC occurs through reference forms issued by dentist-surgeons at the BHU. A study reveals that in $85,7 \%$ of the specialized clinics in the State of Paraná, the access with referral from the $\mathrm{BHU}$ predominates ${ }^{30}$.

This DSC is included in $87,8 \%$ of the facilities which perform the counter reference ${ }^{30}$. Hence, in the end of the specialized procedures, the user is referred to $\mathrm{BC}$, in an organized counter reference system, as the Guideline of Oral Health Network predicts ${ }^{10}$, and constitutes another important aspect for a good interface and integration among the levels of care. 
The logics of work at the DSC must involve the programmed scheduling of the user, in order to guarantee the continuity in care, aiming to reach the user's discharge or their return to the BHU. In this way, the DSC need to adapt themselves to these logics and contribute with integrality, through the access, the treatment, and return, counter reference of the user to professional who assisted him/her at $\mathrm{BC}^{8}$.

\section{Final considerations}

The present study enabled to broaden the analysis of the role of the DSC in the $20^{\text {th }}$ RHS of Paraná, so that the service shows to be of great importance to strengthen the $\mathrm{OHCN}$, and consequently, the NOHP, on the perspective of materializing the integrality of care. However, the network is not still consolidated since there is the absence of a defined flux to high complexity.

Besides, it must be highlighted that the role of the DSC is still understood only as a unit which carries out specialized assistance. It is also noted that the occurrence of only one implemented DSC in a large region favors the acknowledgement and identification of service, but on the other hand, it makes the access difficult and contributes to the restrained demand. Hence, the importance to broad the access to specialized care is highlighted as well. With that, it will be possible to guarantee an even and qualify access to the oral health needs of the population, with the warranty of continuity of care which was initiated at the BHU.

Regarding the interface with $\mathrm{BC}$, actions which can qualify the demand have to be encouraged. It is believed there should be a greater articulation among the levels of care, in order to improve the reference and counter reference fluxes, the development and agreement of clinical protocols, more effective matrix support, and with case discussion, so that there will be a better classification of the role of the DSC.

The fragility of this study which is the absence of quantitative data must be emphasized as to analyze the performance of this DSC through the indicator Global Fulfillment of Goals (GFG), for example. Moreover, there is the need of research that could uncover the user's point of view about the service, with well-deserved consideration.

\section{Reference}

1. Ministry of Health of Brazil. Department of Primary Care, Health Care Secretariat. [SB Brazil Project 2003. Oral health conditions of the Brazilian population, 2002-2003: main results]. Brasilia: Ministry of Health; 2004. Portuguese.

2. Pucca GA Jr, Gabriel M, de Araujo ME, de Almeida FC. Ten Years of a National Oral Health Policy in Brazil: Innovation, Boldness, and Numerous Challenges. J Dent Res. 2015 Oct;94(10):1333-7. doi: $10.1177 / 0022034515599979$.

3. Figueiredo N, Goes PS. [Development of secondary dental care: a study on specialized dental clinics in Pernambuco State, Brazil]. Cad Saude Publica. 2009 Feb;25(2):259-67. doi: 10.1590/s0102-311×2009000200004. Portuguese.

4. Ministry of Health of Brazil. [National Oral Health Policy Guidelines]. Brasília: Ministry of Health; 2004. Portuguese. 
5. Narvai PC. [Collective oral health: ways from sanitary dentistry to buccality]. Rev Saude Publica. 2006 Aug;40 Spec no.:141-7. doi: 10.1590/s0034-89102006000400019. Portuguese.

6. Costa JFR, Chagas LD, Silvestre RM, organizers. [The National Oral Health Policy of Brazil: record of a historic achievement]. Brasilia: Pan American Health Organization, Ministry of Health of Brazil; 2006. Portuguese.

7. Ministry of Health of Brazil. Health Care Secretariat. Department of Primary Care. [Oral health. Primary Care Notebook n 17]. Brasilia: Ministry of Health; 2006. Portuguese.

8. Silva MAM, Silva NA, Zina L, Werneck MAF. [The importance of management actions in the organization of work for CEOs]. In: Figueiredo N, Goes PSA, Martelli P. [The paths of oral health in Brazil: A quanti and quali look on the Centers of Dental Specialties (CEO) in Brazil]. Recife: UFPE publishing; 2016. p.100-38. Portuguese.

9. Ministry of Health of Brazil. [Oral health in the Unified Health System]. Brasilia: Ministry of Health; 2018. Portuguese.

10. Paraná State Department of Health. Health Care Superintendence. [Oral health guide line]. Curitiba: SESA; 2016. Portuguese.

11. Lucena EHG, Sobrinho JEL, Werneck MAF, Martelli PJL, Oliveira TF. [Implementation of Dental Specialization Centers in the regionalization process of oral health care in Brazil]. In: Figueiredo N, Goes PSA, Martelli P. [The paths of oral health in Brazil: A quanti and quali look on the Centers of Dental Specialties (CEO) in Brazill. Recife: UFPE publishing; 2016. p.27-36. Portuguese.

12. Brazil. [Ordinance No. 4,279. Establishes guidelines for the organization of the Health Care Network within the scope of the Unified Health System (SUS)]. Brasilia: Official Gazette of the Federative Republic of Brazil; 2010 Dec 30. Portuguese.

13. Pinto MHB, Araújo ME, Fujimaki M, Ditterich RG, Terada RSS, Martelli PJL. [The Oral Health Care Networks - The role of the CEO]. In: Figueiredo N, Goes PSA, Martelli P. [The paths of oral health in Brazil: A quanti and quali look on the Centers of Dental Specialties (CEO) in Brazil]. Recife: UFPE publishing; 2016. p.221-35. Portuguese.

14. Ditterich RG, Moysés SJ, Graziani GF, Couto MP, Bueno RE, Gabardo MCL. [Building oral health paths in the state of Paraná]. In: Ditterich RG, Graziani GF, Moisés SJ, organizers. [Paths and trajectories of oral health in the state of Paraná]. Londrina: SESA/INESCO; 2019. p.15-37. Portuguese.

15. Santiago BM, Amaral JHL, Zina LG, Werneck MAF, Padilha WWN. [The University, the CEO and the PMAQ-CEO]. In: Figueiredo N, Goes PSA, Martelli P. [The paths of oral health in Brazil: A quanti and quali look on the Centers of Dental Specialties (CEO) in Brazil]. Recife: UFPE publishing; 2016. p. 49-62. Portuguese.

16. Paraná State Department of Health. [Regionalization Master Plan]. Curitiba: SESA; 2015. 214p. Portuguese.

17. State Secretariat of Planning and Structuring Projects of Paraná. Instituto Paraná of Economic and Social Development - IPARDES. [Demographic and Social Base]. [cited 2020 Jul 12]. Available from: http://www.ipardes.gov.br. Portuguese.

18. Martins J, Bicudo MAV. [Qualitative research in psychology: fundamentals and basic resources]. São Paulo: Centauro; 2005. Portuguese.

19. de Mello AL, de Andrade SR, Moysés SJ, Erdmann AL. [Oral health care in the health network and the regionalization process]. Cien Saude Colet. 2014 Jan;19(1):205-14. doi: 10.1590/141381232014191.1748. Portuguese.

20. Silva CR, Pinafo E. [Northern macro-region of Paraná: demographic, socioeconomic and epidemiological situation of the municipalities]. In: Carvalho BG, Nunes EFPA, Cordoni Junior L, organizers. [Health management in small municipalities: the case of northern Paraná]. Londrina: Eduel; 2018. p.40-63. Portuguese. 
21. Gonçalves JRS, Ditterich RG, Gonçalves RN, Gavina VP, Silva ROC, Kusma SZ. [Performance of Dental Care Specialties Centers of the 2nd Health Regional from Paraná]. Cad Saude Colet. 2018;26(4):432-8. doi: 10.1590/1414-462×201800040429. Portuguese.

22. Goes PS, Figueiredo N, Neves JC, Silveira FM, Costa JF, Pucca Júnior GA, et al. [Evaluation of secondary care in oral health: a study of specialty clinics in Brazil]. Cad Saude Publica. 2012;28 Suppl:s81-9. doi: 10.1590/s0102-311×2012001300009. Portuguese.

23. Thomaz EB, Sousa GM, Queiroz RC, Coimbra LC. [Evaluating productivity targets achievement in Dental Specialty Centers from Maranhão State, Brazil, 2011]. Epidemiol Serv Saude. 2016 Oct-Dec;25(4):807-18. doi: 10.5123/S1679-49742016000400014. Portuguese.

24. Figueiredo N, Goes PSA, Martelli PJL, organizers. [Report of the 1st cycle of the external evaluation of the Access and Quality Improvement Program of the Dental Specialties Centers (AVE-PMAQ / CEO): main results]. Recife: Editora UFPE Publisher; 2016. Portuguese.

25. Paraná State Department of Health. [Oral Health Care Network]. 2016[cited 2020 Jul 12]. Available from: http://www.saude.pr.gov.br/modules/conteudo/conteudo.php?conteudo=3027. Portuguese.

26. Lavras C. [Primary health care and the organization of regional health care networks in Brazil]. Saúde Soc. 2011;20(4):867-74. doi: 10.1590/S0104-12902011000400005. Portuguese.

27. Mendes, EV. [Health care networks]. 2. ed. Brasilia: Pan American Health Organization; 2011. Portuguese.

28. Chaves SCL, Barros SG, Cruz DN, Figueiredo AC, Moura BLA, Cangussu MCT. Brazilian Oral Health Policy: factors associated with comprehensiveness in health care. Rev Saude Publica. 2010;44(6):1005-13. doi: 10.1590/S0034-89102010005000041.

29. Aquilante AG, Aciole GG. Oral health care after the National Policy on Oral Health - "Smiling Brazil": a case study. Cienc Saude Colet. 2015;20(1):239-48. doi: 10.1590/1413-81232014201.21192013.

30. Gonçalves JRS, Moysés SJ, Ditterich RG, Terada RSS, Gonçalves RN. [The Dental Specialties Centers (CEO) as a point of attention in the oral health network in Paraná: an analysis of the PMAQ-CEO results]. In:Ditterich RG, Graziani GF, Moisés SJ, organizers. [Paths and trajectories of oral health in the state of Paraná]. Londrina: SESA/INESCO; 2019. p.197-212. Portuguese. 